\title{
Partial ALPPS with a longer wait between procedures is safe and yields adequate future liver remnant hypertrophy
}

\author{
Nagappan Kumar ${ }^{1}$, Trish Duncan ${ }^{1}$, David O'Reilly ${ }^{1}$, Zsolt Káposztás ${ }^{2}$, \\ Craig Parry ${ }^{3}$, John Rees ${ }^{3}$, and Sameer Junnarkar ${ }^{4}$
}

${ }^{1}$ Cardiff Liver Unit, University Hospital of Wales, Cardiff, UK, ${ }^{2}$ Moritz Kaposi Teaching General Hospital, Kaposvár, Hungary, ${ }^{3}$ Department of Radiology, University Hospital of Wales, Cardiff, UK, ${ }^{4}$ Department of Surgery, Tan Tock Seng Hospital, Singapore

\begin{abstract}
Backgrounds/Aims: Associating Liver Partition and Portal Vein Ligation for Staged Hepatectomy (ALPPS) has generated controversy due to high morbidity and mortality. We present our series of patients with $30-40 \%$ parenchymal transection and minimal hilar dissection. Methods: Patients who had partial ALPPS between April 2015 and April 2016 were included. Patients with colorectal liver metastases (CRLM) had their future liver remnants (FLR) cleared with metastasectomies. The liver was divided along the future line of transection to $30-40 \%$, right portal vein was stapled and divided without extensive hilar dissection, with minimal handling of right liver, which was not mobilised. We preserved the middle hepatic vein. Data were collected prospectively for hypertrophy of the FLR, morbidity and mortality. Results: Among the 8 patients (age 25-68) investigated, one patient with cholangiocarcinoma had portal vein embolization prior to partial ALPPS. All patients completed two stages with adequate FLR hypertrophy at a median of 28 days. No mortality was found. The median length of stay after stages 1 and 2 was 9 and 9.6 days, respectively. The median increase in FLR was $38 \%$. Conclusions: A limited transection of $30-40 \%$, minimal hilar dissection and longer wait between stages yielded adequate FLR hypertrophy with low morbidity and no mortality. (Ann Hepatobiliary Pancreat Surg 2019;23:13-19)
\end{abstract}

Key Words: Liver resection; Future liver remnant; ALPPS; Hypertrophy; Colorectal liver metastases

\section{INTRODUCTION}

Liver resection offers the best chance for long-term survival of patients with colorectal liver metastases (CRLM) and hilar cholangiocarcinoma. A combination of better chemotherapeutic regimens and surgical innovations has increased the resection rates in patients with CRLM. Several technical limiting factors such as location of the tumor and distribution preclude liver resection. A future liver remnant (FLR) of $20-30 \%$ is deemed safe in patients with normal background liver and at least $40 \%$ FLR in patients with compromised liver (steatohepatitis, fibrosis, cirrhosis). ${ }^{1}$ The techniques that have proved successful in increasing resection rates include neoadjuvant chemotherapy in patients with CRLM, ${ }^{2-4}$ portal vein embolization (PVE), portal vein ligation (PVL), ${ }^{6}$ two-stage liver resections, ${ }^{7}$
Associating Liver Partition and Portal vein ligation in Staged hepatectomy (ALPPS) ${ }^{8}$ and Associating Portal Embolization and Artery Ligation (APEAL). ${ }^{9}$

The ALPPS procedure created tremendous interest among liver surgeons because of the pace of liver regeneration that it allowed, enabling completion of the two stages of liver resection within 9 to 11 days. The procedure has yet to be widely adopted mainly due to concerns with increasing morbidity, mortality ${ }^{10}$ and potentially higher recurrence rates. $^{11,12}$

We performed the first ALPPS procedure in April 2015 using only partial transection of liver and other technical modifications involving minimal dissection. This approach produced significant hypertrophy of the FLR and we have performed 8 procedures with no mortality and acceptable morbidity. We proceeded to the second stage in all pa-

Received: June 14, 2018; Revised: July 23, 2018; Accepted: July 26, 2018

Corresponding author: Nagappan Kumar

Cardiff Liver Unit, University Hospital of Wales, Cardiff, CF144XW, UK

Tel: +44-29-2074-4793, Fax: +44-29-2074-3348, E-mail: nagappan.kumar@wales.nhs.uk

Copyright (C) 2019 by The Korean Association of Hepato-Biliary-Pancreatic Surgery

This is an Open Access article distributed under the terms of the Creative Commons Attribution Non-Commercial License (http://creativecommons.org/ censes/by-nc/4.0) which permits unrestricted non-commercial use, distribution, and reproduction in any medium, provided the original work is properly cited. Annals of Hepato-Biliary-Pancreatic Surgery - pISSN: 2508-5778 - elSSN: 2508-5859 
tients after a longer interval than the classical ALPPS. The aim of this report is to show the feasibility of safe two-stage liver resection via transection of only 30-40\% of liver (partial ALPPS). A longer interval between the two procedures may lead to less liver failure.

\section{PATIENTS AND METHODS}

In order to describe the modified version of the ALPPS procedure, the first operation is briefly explained below.

The patient was a 68-year-old lady with obstructing carcinoma of descending colon and synchronous liver metastases. She underwent left hemicolectomy with end stoma in January 2014 for a T4bN0M1 tumor. The liver metastases involved all segments except segment 1 and 5 (Fig. 1). The patient had palliative chemotherapy with 4 cycles of XELOX (capecitabine plus oxaliplatin) following progression with FOLFIRI (leucovorin calcium, 5-fluorouracil, and irinotecan) regimen as second-line treatment. Aflibercept was added to augment the response, and was completed in February 2015. An Fludeoxyglucose 18F (FDG)-positron emission tomography (PET) scan revealed liver only metastases and a pair of foci in the left lateral abdominal wall suggestive of local recurrence. However, review of cross-sectional images in this area revealed no masses and after discussion with the hepatobiliary multidisciplinary team (MDT) meeting it was decided to explore the possibility of two-stage liver resection.

Using laparotomy, the tumors in the segments 2, 3 and $4 \mathrm{~b}$ were removed as metastasectomies (Fig. 2) with macroscopically clear margins. The FLR was inadequate. Cholecystectomy was carried out and the right portal vein (RPV) was ligated with minimal dissection by incising the peritoneum on the right and posterior aspects of the hilum. The RPV was stapled and divided (Fig. 3). Metastasectomy of the tumor in the segment $4 \mathrm{~b}$ led to a large defect in the liver with only a small sliver of tissue between this defect and the gall bladder fossa. This was divided down to the hilum resulting in transection of nearly a third of liver. The right liver was not mobilized. A silastic drain
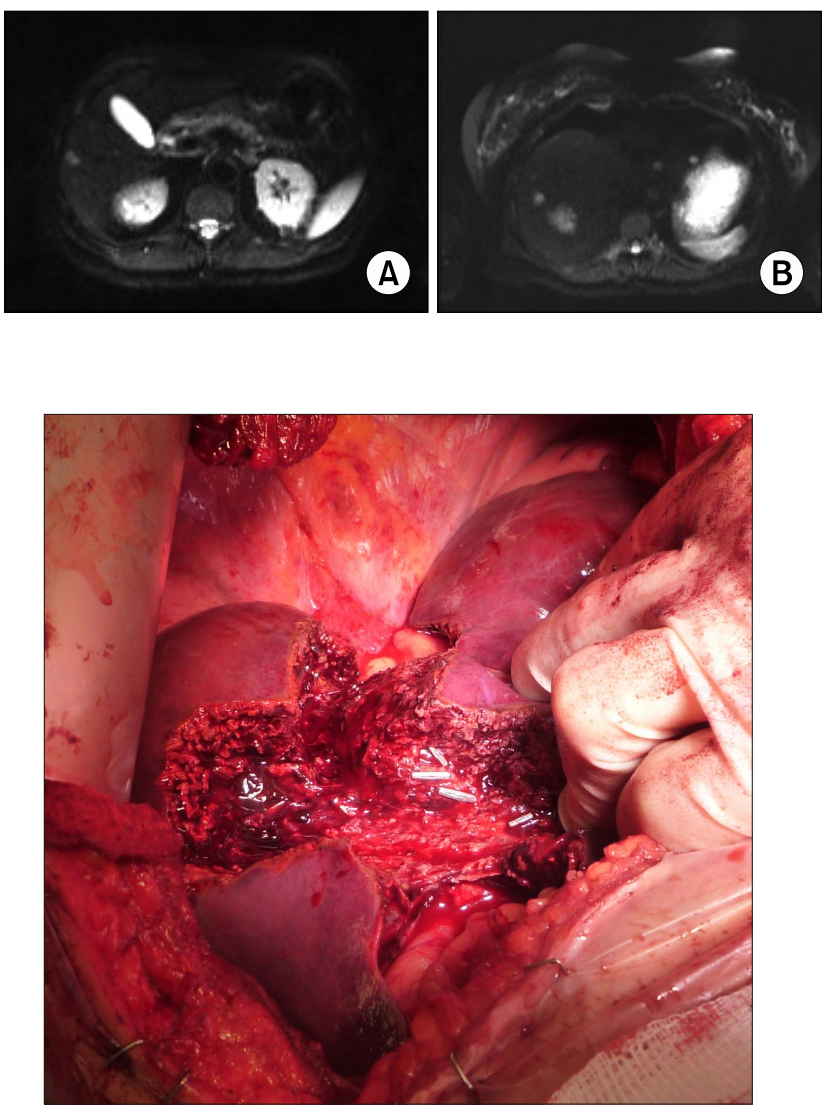

Fig. 2. Intraoperative photograph showing metastasectomies in segment 4.

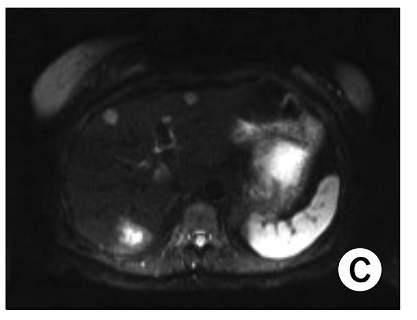

Fig. 1. Metastases detected in the hepatic segment 6 (A), segments 2 , and 7 (B), segments 3 , 4 , and 8 (C).

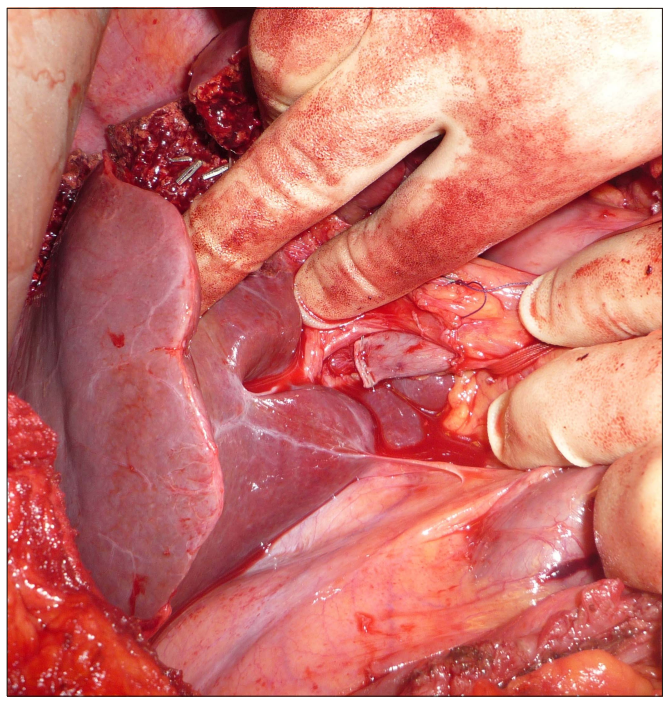

Fig. 3. Right portal vein stapling (arrow) with minimal hilar dissection. 
was placed along the cut surface of the liver. The patient recovered adequately and was discharged on postoperative day 6 with the drain in situ. Histological examination revealed complete resection with a margin of at least $1 \mathrm{~mm}$. A computed tomography (CT) scan was performed to measure the volume of the FLR 12 days after the first stage procedure and volumes were assessed using standard procedure described below. ${ }^{13}$

The CT assessment showed an increase in FLR from $408 \mathrm{ml}$ to $630 \mathrm{ml}$, or an increase of $54 \%$. She then underwent a completely extended right hemihepatectomy 21 days after the first stage procedure, using and completing the previous transection plane. Histologically, the tumor in the segment 7 close to the inferior vena cava extended to within $1 \mathrm{~mm}$ of the resection margin. The patient recovered without any complication and was discharged 7 days after the operation.

The above experience indicated that FLR hypertrophy was feasible with partial transection of the liver. We performed similar procedures in seven other patients reported here. We defined $30-40 \%$ transection for an extended right hepatectomy as resection of the liver along the falciform ligament and removing the segment $4 \mathrm{~b}$ pedicle. The cut was extended up to the segment 4 a pedicle which was left in situ. The middle hepatic vein was not divided in the first operation.

\section{Liver volume assessment}

Liver volume was assessed using commercial volumetry software, Volume Viewer advanced imaging platform (GE Healthcare Version 2.0, AW Server) and calculated by manually defining the contours of the liver parenchyma on representative sequential axial $0.625 \mathrm{~mm}$ slices of the CT acquisition on a liquid crystal display $(1920 \times 1200$ resolution, 24-inch wide screen display, LA2405, HP Compaq).

The volume rendering tool automatically generates a $3 \mathrm{D}$ volume and calculates the total liver volume (TLV) from the selected slices. This process was repeated for all definable liver lesions to yield the estimated total lesion volume and the two volumes subtracted to determine the estimated Total Functional Liver Volume (TFLV).

The selected liver volume was used to define the predicted Functional Residual Liver Volume (FRLV) by segmenting and excluding the hepatic parenchyma planned for resection as directed by the liver surgeon. The ratio of predicted functional residual liver volume (\%FRLV) was then calculated.

The actual FRLV was subsequently measured on the post-ALPPS axial CT performed on day 14 to determine the extent of hypertrophy and feasibility of complete hepatectomy.

\section{Hepatobiliary (HIDA) assessment}

We performed a HIDA scan as part of the evaluation following the first stage for differential evaluation of the function of FLR. Hepatobiliary scintigraphy was performed by injecting $70 \mathrm{MBq}$ of ${ }^{99 \mathrm{~m}} \mathrm{Tc}$-mebrofenin intravenously after a 4-h fast. Dynamic anterior and posterior images were acquired for $60 \mathrm{~min}$, with the patient lying supine on a large field-of-view dual-headed gamma camera (Millennium Hawkeye, GE) equipped with low-energy high-resolution collimators. Images were grouped into twelve 5-minute frames. The images were assessed, both to calculate the function of the future liver remnant, and also to exclude biliary leak.

Quantitation of the FLR was performed by drawing regions of interest around the left and right liver on both the anterior and posterior 0-5-min grouped image, as defined by the prior hepatic cut. The \% contribution of each half of the liver was calculated by geometric mean.

In summary, all patients with CRLM had a CT chest, abdomen and pelvis, magnetic resonance imaging of the liver and a FDG PET scan before surgery. The patients were discharged after the first stage and exposed to a CT scan of abdomen and a HIDA scan 2 weeks from the procedure. The second stage procedure was scheduled 3 weeks from first stage. Chemotherapy was completed at least 6 weeks before the first stage procedure in all patients with CRLM.

We recorded the complications and classified them according to the Clavien-Dindo system. ${ }^{14}$ Post-hepatectomy liver failure (PHLF) was classified according to the grades proposed by the International Study Group of Liver Surgery. ${ }^{15}$

We followed up patients at 4 weeks after surgery and at 3 monthly intervals. The CT scan of chest abdomen and pelvis was carried out at 3 months, 6 months and a year from the second stage procedure. Recurrence data and mortality were censored at December 2016. 
The procedure was approved by the Clinical Effectiveness Committee at the University Hospital of Wales (UK).

\section{RESULTS}

A total of 8 patients, with a median age of 61 years (25-68), 6 with CRLM and 2 with hilar cholangiocarcinoma were studied. All patients proceeded to a second stage operation with adequate FLR hypertrophy at a median interval of 32 (21-36) days between the procedures. No mortality was detected. The pre and post mini-ALPPS FLR, morbidity and mortality data are presented in Table 1 .

The last patient had a pre-operative PVE and a trial dissection for a hilar cholangiocarcinoma. The liver volume was inadequate at the time of surgery despite a reasonable response to PVE and a partial parenchymal transection was performed. This patient developed post-operative bile leak requiring surgical washout. Following recovery, the second-stage resection was performed on day 36. Otherwise, following first-stage resection, 1 patient developed a bile leak and another patient presented with hospital-acquired pneumonia. Following second-stage resection, 1 patient developed transient liver failure and 2 patients developed bile leaks (1 requiring endoscopic retrograde cholangiopancreatography). All of the patients underwent CT scan at a median of 14 days (11-35) of first-stage surgery to assess the volume of the FLR. A hypertrophy of $10 \%$ to $78 \%$ was achieved. Second-stage operations included right trisectionectomies. Patients were followed up for a median of 390 days (233-472).

\section{DISCUSSION}

We have shown that FLR can hypertrophy enough to allow second-stage resection by only dividing the liver partially. Besides, other techniques listed below were used to reduce morbidity and mortality. The right liver which was scheduled for resection in the second stage was not mobilized at all. The hilar dissection only involved an incision of the peritoneal layer on the right and posterior aspects of the free edge of the lesser omentum to allow access to the right portal vein. This was divided using a stapler. No bag was used to isolate the liver for subsequent resection, thus reducing the risk of infection. A silastic drain was left where the liver was divided to en-

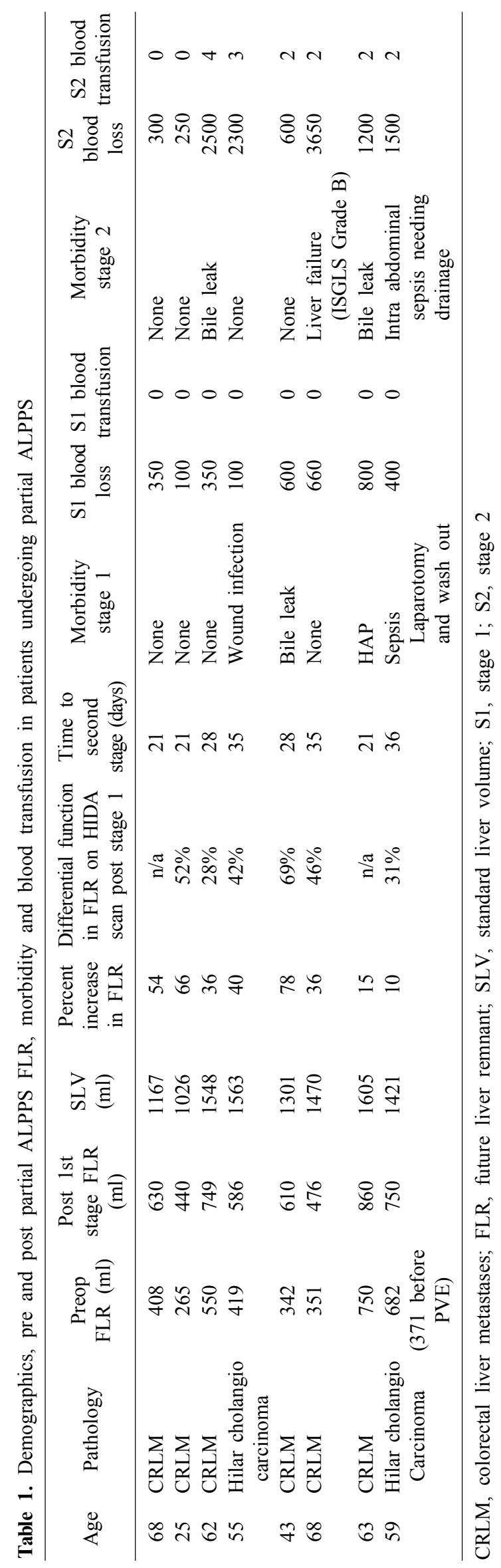


able easy access to the second procedure. We left a long polydioxanone (PDS) suture around the hilum as a loop for easy identification of the hilum to facilitate Pringle's maneuver during the second operation.

de Santibañes et al. ${ }^{16}$ have described a similar procedure in four patients. However, they did not perform any hilar dissection during the first stage. Instead, they performed intraoperative PVE using the inferior mesenteric vein and designated the procedure as Mini-ALPPS. They claim that the lack of hilar dissection is beneficial for the second stage. We perform minimal hilar dissection and only the right and posterior peritoneum on the hilum is dissected to allow access to the right portal vein, which is stapled. We have not encountered any difficulties related to this during the second stage. We started to perform the procedure before the Santibanes paper was published, and hence our approach of minimal hilar dissection. This approach also avoids any potential logistic difficulties in performing PVE in the theatre. Li et al. ${ }^{17}$ described similar operation of PVE 2 days after the first procedure in patients with tumor involving the hilum.

The advantages of ALPPS include the higher completion rates of the two stages compared with two-stage hepatectomies (TSH). However, critics have shown a poorer long-term outcome in patients undergoing ALPPS compared with $\mathrm{TSH}^{18}{ }^{18}$ Our study is an observational study and hence prone to many biases. A randomized controlled trial is the only way to compare the long-term outcomes of ALPPS vs. TSH. Although no randomized controlled trial compared ALPPS and TSH, a systematic review and meta-analysis of all the comparative studies showed that the overall survival was not different between the two approaches. ALPPS was associated with higher morbidity and mortality. ${ }^{19}$ In addition, higher rates of liver failure followed ALPPS despite volumetric increase in the remnant liver. Matsuo et al. have shown that the hepatocytes that regenerate after ALPPS were morphologically immature compared with PVE. ${ }^{20}$ Only one patient developed ISGLS grade B liver failure in our series, with a 35-day interval between the procedures. The median interval between the two procedures was 21 days and 5 out of the 8 patients underwent the second procedure within 30 days of the first intervention. This longer interval compared with the 9-11 days described in most series may have reduced the liver failure, which requires confirmation in a larger series or a randomized controlled trial. The last two patients in the series did not manifest significant hypertrophy ( $15 \%$ and $10 \%$ respectively), which is most likely due to portoportal collaterals found on the post-partial ALPPS CT scan. The existence or development of portoportal collaterals is a significant factor impeding hypertrophy following $\mathrm{PVE}^{21}$

The median increase in hypertrophy in our patients was $38 \%(10-78 \%$ ), which was lower than in classical ALPPS, where the range varied from $58 \%$ to $110 \%{ }^{22}$ and attributed to unidentified portoportal collaterals. Indeed the mechanism for significant hypertrophy following ALPPS is not fully understood. Our technique yielded enough hypertrophy such that all patients proceeded to the second stage operation.

It could be argued that the initial liver volumes were adequate to perform a single-stage procedure. However, we preferred two stages based on the quality of the liver at the time of the first stage along with the volume. Røsok et al. $^{23}$ reported the Scandinavian experience involving a small number of patients, who were converted to ALPPS during a planned single-stage operation. The factors underlying the decision included detection of additional lesions or suspected poor quality of liver, which was critiqued by Belghiti et al. ${ }^{24}$ in their editorial in the same issue of the journal, suggesting that many of these patients could have been operated with a single-stage procedure. Liver failure after resection carries high mortality. Currently, except for the volume, no predictors are available for the development of liver failure. However, Cieslak et al. ${ }^{25}$ showed that ${ }^{99 \mathrm{~m}} \mathrm{Tc}$-mebrofenin scintigraphy with a cut-off value of $2.7 / \mathrm{min} / \mathrm{m}^{2}$ may be used to assess adequacy of FLR function. Further, no strict correlation with increase in volume was observed with CT volumetry. The HIDA scan was not used in the same way. The differential volume of the liver was calculated and in the presence of adequate FLR (i.e. $>25 \%$ ) we proceeded to the second stage of ALPPS.

We have shown that partial ALPPS is a safe alternative to the ALPPS originally described. It facilitates safe and rapid two-stage procedure for tumor clearance in patients with CRLM. Many previous studies report a near 100\% success in proceeding to the second stage. However, subsequent to this report we found a 79-year-old patient who failed to proceed to second-stage ALPPS due to a heart 
block and was thus contraindicated for the procedure. The patient lacked adequate hypertrophy of the FLR and underwent PVE due to abnormal anatomy that allowed perfusion of the right liver. The long-term outcomes in patients undergoing ALPPS for CRLM are questionable as well.

There are several limitations to our study. This is a small study of only 8 patients, and the technique used to measure volumes by HIDA scan was not validated. It is possible that the recorded volumes may have been adequate for a single-stage procedure. We feel that the safety of the procedure facilitates liver surgeons to consider this option that is intermediate between traditional ALPPS and TSH. A longer interval between the two procedures along with the use of ${ }^{99 \mathrm{~m}}$ Tc-mebrofenin scintigraphy to assess FLR function may prevent postoperative liver failure.

This preliminary experience may allow prospective consideration of interventions in patients requiring FLR clearance that is inadequate without an additional procedure such as PVE or PVL. Modifications to the original ALPPS described facilitate safe two-stage hepatectomy, mainly in patients with CRLM.

\section{REFERENCES}

1. Adams RB, Aloia TA, Loyer E, Pawlik TM, Taouli B, Vauthey JN. Selection for hepatic resection of colorectal liver metastases: expert consensus statement. HPB (Oxford) 2013;15:91-103.

2. Bismuth H, Adam R, Lévi F, Farabos C, Waechter F, Castaing $\mathrm{D}$, et al. Resection of nonresectable liver metastases from colorectal cancer after neoadjuvant chemotherapy. Ann Surg 1996; 224:509-520; discussion 520-522.

3. Folprecht G, Gruenberger T, Bechstein WO, Raab HR, Lordick F, Hartmann JT, et al. Tumour response and secondary resectability of colorectal liver metastases following neoadjuvant chemotherapy with cetuximab: the CELIM randomised phase 2 trial. Lancet Oncol 2010;11:38-47.

4. Lam VW, Spiro C, Laurence JM, Johnston E, Hollands MJ, Pleass $\mathrm{HC}$, et al. A systematic review of clinical response and survival outcomes of downsizing systemic chemotherapy and rescue liver surgery in patients with initially unresectable colorectal liver metastases. Ann Surg Oncol 2012;19:1292-1301.

5. van Lienden KP, van den Esschert JW, de Graaf W, Bipat S, Lameris JS, van Gulik TM, et al. Portal vein embolization before liver resection: a systematic review. Cardiovasc Intervent Radiol 2013;36:25-34.

6. Capussotti L, Muratore A, Baracchi F, Lelong B, Ferrero A, Regge D, et al. Portal vein ligation as an efficient method of increasing the future liver remnant volume in the surgical treatment of colorectal metastases. Arch Surg 2008;143:978-982; discussion 982.

7. Adam R, Laurent A, Azoulay D, Castaing D, Bismuth $\mathrm{H}$. Two-stage hepatectomy: a planned strategy to treat irresectable liver tumors. Ann Surg 2000;232:777-785.

8. Schnitzbauer AA, Lang SA, Goessmann H, Nadalin S, Baumgart J, Farkas SA, et al. Right portal vein ligation combined with in situ splitting induces rapid left lateral liver lobe hypertrophy enabling 2-staged extended right hepatic resection in small-for-size settings. Ann Surg 2012;255:405-414.

9. Dupré A, Hitier M, Peyrat P, Chen Y, Meeus P, Rivoire M. Associating portal embolization and artery ligation to induce rapid liver regeneration in staged hepatectomy. Br J Surg 2015;102: 1541-1550.

10. Schadde E, Raptis DA, Schnitzbauer AA, Ardiles V, Tschuor C, Lesurtel M, et al. Prediction of mortality after ALPPS stage-1: an analysis of 320 patients from the International ALPPS Registry. Ann Surg 2015;262:780-785; discussion 785-786.

11. Figueras J, Belghiti J. The ALPPS approach: should we sacrifice basic therapeutic rules in the name of innovation? World J Surg 2014;38:1520-1521.

12. Oldhafer KJ, Donati M, Jenner RM, Stang A, Stavrou GA. ALPPS for patients with colorectal liver metastases: effective liver hypertrophy, but early tumor recurrence. World J Surg 2014; 38:1504-1509.

13. Urata K, Kawasaki S, Matsunami H, Hashikura Y, Ikegami T, Ishizone $\mathrm{S}$, et al. Calculation of child and adult standard liver volume for liver transplantation. Hepatology 1995;21:1317-1321.

14. Dindo D, Demartines N, Clavien PA. Classification of surgical complications: a new proposal with evaluation in a cohort of 6336 patients and results of a survey. Ann Surg 2004;240:205-213.

15. Rahbari NN, Garden OJ, Padbury R, Brooke-Smith M, Crawford M, Adam R, et al. Posthepatectomy liver failure: a definition and grading by the International Study Group of Liver Surgery (ISGLS). Surgery 2011;149:713-724.

16. de Santibañes E, Alvarez FA, Ardiles V, Pekolj J, de Santibañes M. Inverting the ALPPS paradigm by minimizing first stage impact: the Mini-ALPPS technique. Langenbecks Arch Surg 2016;401:557-563.

17. Li J, Kantas A, Ittrich H, Koops A, Achilles EG, Fischer L, et al. Avoid "all-touch" by hybrid ALPPS to achieve oncological efficacy. Ann Surg 2016;263:e6-e7.

18. Adam R, Imai K, Castro Benitez C, Allard MA, Vibert E, Sa Cunha A, et al. Outcome after associating liver partition and portal vein ligation for staged hepatectomy and conventional twostage hepatectomy for colorectal liver metastases. Br J Surg 2016;103:1521-1529.

19. Moris D, Ronnekleiv-Kelly S, Kostakis ID, Tsilimigras DI, Beal EW, Papalampros A, et al. Operative results and oncologic outcomes of Associating Liver Partition and Portal Vein Ligation for Staged Hepatectomy (ALPPS) versus Two-Stage Hepatectomy (TSH) in patients with unresectable colorectal liver metastases: a systematic review and meta-analysis. World J Surg 2018;42: 806-815.

20. Matsuo K, Murakami T, Kawaguchi D, Hiroshima Y, Koda K, Yamazaki $\mathrm{K}$, et al. Histologic features after surgery associating liver partition and portal vein ligation for staged hepatectomy versus those after hepatectomy with portal vein embolization. Surgery 2016;159:1289-1298.

21. Zeile M, Bakal A, Volkmer JE, Stavrou GA, Dautel P, Hoeltje $\mathrm{J}$, et al. Identification of cofactors influencing hypertrophy of the future liver remnant after portal vein embolization-the effect of collaterals on embolized liver volume. Br J Radiol 2016;89: 20160306.

22. Schadde E, Schnitzbauer AA, Tschuor C, Raptis DA, Bechstein WO, Clavien PA. Systematic review and meta-analysis of feasibility, safety, and efficacy of a novel procedure: associating liver partition and portal vein ligation for staged hepatectomy. Ann 
Surg Oncol 2015;22:3109-3120.

23. Røsok BI, Björnsson B, Sparrelid E, Hasselgren K, Pomianowska E, Gasslander T, et al. Scandinavian multicenter study on the safety and feasibility of the associating liver partition and portal vein ligation for staged hepatectomy procedure. Surgery 2016; 159:1279-1286.

24. Belghiti J, Dokmak S, Schadde E. ALPPS: innovation for in- novation's sake. Surgery 2016;159:1287-1288.

25. Cieslak KP, Olthof PB, van Lienden KP, Besselink MG, Busch OR, van Gulik TM, et al. Assessment of liver function using (99m)Tc-mebrofenin hepatobiliary scintigraphy in ALPPS (Associating Liver Partition and Portal Vein Ligation for Staged Hepatectomy). Case Rep Gastroenterol 2015;9:353-360. 